\title{
Remue-méninges dans les sciences du genre
}

\author{
Entretien avec Rebecca Jordan-Young \\ Mené et traduit par $\overline{\text { Vanina Mozziconacci }{ }^{1}}$
}

\begin{abstract}
Rebecca Jordan-Young est chercheuse en sciences sociomédicales, un programme interdisciplinaire qui réunit les théories et méthodes des sciences sociales et des sciences de la santé, et s'est spécialisée dans l'analyse des dispositifs de mesure et des protocoles d'étude. Elle a une chaire en Women's, Gender, and Sexuality Studies au Barnard College, rattaché à l'Université Columbia, où elle est la directrice du Science and Social Difference Working Group. Son travail se situe à l'intersection de la science, du genre, de la sexualité et de la «race». Son ouvrage paru en 2010, Brain Storm : The Flaws in the Science of Sex Differences², est le résultat de treize années d'analyse des études fondées sur la «théorie organisationnelle » du cerveau humain, selon laquelle l'exposition aux hormones pendant la période prénatale est à l'origine d'une différenciation sexuée du cerveau. Un examen approfondi de plus de trois cents études ainsi que des entretiens avec plus de vingt scientifiques lui ont permis de livrer une analyse méticuleuse des biais et des contradictions de ce champ de recherche. Nous l'avons rencontrée lors d'un séjour de recherche à l'Université Columbia durant l'automne 2014 afin d'échanger à propos de son livre.
\end{abstract}

Vous qualifiez votre position scientifique d'« hybride » car votre travail est influencé par les laboratory studies en ethnographie ${ }^{3}$ mais contrairement à elles, vous évaluez la « science en train de se faire » à l'aune d'un idéal scientifique. Cependant, votre idéal n'est pas celui d'une science purgée des facteurs sociaux qui la «contaminent ». Votre approche est-elle liée à une épistémologie féministe?

\footnotetext{
${ }^{1}$ Je remercie chaleureusement Rebecca Jordan-Young pour sa collaboration et Odile Fillod pour sa relecture. Toute ma gratitude va également à l'AFFDU/ Dorothy Leet et au Programme Avenir Lyon Saint-Étienne pour leur soutien.

${ }^{2}$ Toutes les références et les notes sont des ajouts de Mozziconacci.

${ }^{3}$ II s'agit d'une partie importante des Science and Technology Studies (STS), qui étudient les conditions sociales de la recherche scientifique. Pour une publication emblématique du domaine, voir Latour et Wolgar 1998.
} 
J'utilise l'épistémologie féministe, assurément. Helen Longino est l'une des philosophes féministes qui m’influence (Longino, 1990 et Longino, 2013). Son épistémologie pragmatique montre que des études scientifiquement solides peuvent appréhender le même objet avec des orientations disciplinaires et des méthodes différentes et se retrouver avec des connaissances qui se contredisent. La science n'est donc pas simplement un ensemble d'outils destinés à révéler des réalités fondamentales que nous pourrions connaître avec une certitude absolue. Plutôt que d'en rester à une telle vision de la science, nous devrions considérer qu'elle est une façon particulièrement systématique d'explorer le monde, dans laquelle nous utilisons une combinaison de nos données sensorielles et de notre capacité à raisonner afin d'évaluer des idées, mais qu'elle contiendra toujours des éléments sociaux. Elle sera toujours orientée par ce que nous pensons savoir avant même de commencer. Helen Longino dit que, puisque la science n'est pas simplement "une», nous devons développer des méthodes supplémentaires de façon à déterminer quelle est la meilleure science pour la question qui nous préoccupe. Les résultats scientifiques ne sont pas inéluctables et n'attendent pas dans la nature d'être découverts, ils sont le produit d'un état matériel et social du monde et des connaissances et techniques que nous apportons. Les épistémologies féministes nous ont appris à utiliser différentes façons de connaître : Donna Haraway (Haraway, 1998), avec sa compréhension de la partialité de la connaissance et de la façon dont la technologie intervient comme médiation dans celle-ci ; Sandra Harding (Harding, 1992), à propos de l'épistémologie du point de vue, et d'autres philosophes, qui ne sont pas identifiéees comme féministes, mais qui sont dans la tradition ethnographique, comme Annemarie Mol (Mol, 2002), qui montre l'importance de la pratique scientifique.

Certains domaines hors des sciences quantitatives, en particulier l'anthropologie, ont reconnu que lorsqu'on cherche à comprendre un monde qui n'est pas le sien, une des méthodes pour savoir que croire, pour savoir si une observation ou une source est juste, est la triangulation. Est-ce que les informations qui viennent de diverses sources concordent? Les échecs dans la triangulation, je les utilise non pas pour trouver des «erreurs » ou des inexactitudes en soi, mais pour voir comment la connaissance dépend d'un contexte. Quand la triangulation échoue, il devient possible d'observer comment les auteur.e.s de ces observations (ou d'autres «connaisseuses-eurs » [knowers] du domaine) sont pris·es dans une ontologie particulière, parce que des pratiques différentes font des objets différents4. Dans Brain Storm, j'étais très sceptique vis-à-vis de l'uniformité des termes utilisés [dans la recherche fondée sur la théorie organisationnelle du cerveau] alors qu'ils désignaient des objets fort diffé-

${ }^{4}$ Voir les notions d'enactment et d'intra-action dans les travaux de Karen Barad (Barad, 1998). 
rents. Si les scientifiques prenaient au sérieux ces différences profondes au lieu de les occulter et de les entasser dans une théorie simple du développement, nous nous rapprocherions d'une appréhension réellement scientifique, empirique et ouverte de la psychologie et des corps humains. Quand un phénomène, comme l'« orientation sexuelle » par exemple, n'a pas la même signification dans des situations différentes, cela ne veut pas dire qu'il ne peut pas être étudié scientifiquement - mais nous devons faire attention à l'importance du contexte, et non simplement maintenir qu'il s'agit de la même chose parce que cela permet d'avoir une théorie plus simple. Nous devons nous demander quel type de science fait sens dans notre monde social.

Précisément, dans ce livre vous insistez sur la question de la cohérence ou de l'incohérence entre les différentes études portant sur le rôle des hormones dans la différenciation sexuée du cerveau. Vous vérifiez si leurs résultats, leurs mesures et leurs définitions s'accordent, et vous montrez que ce n'est pas le cas, et qu'ils sont parfois même contradictoires. De plus, vous montrez que ce modèle occulte des aspects importants du développement humain. Diriezvous que ce que vous recherchez dans une théorie, c'est moins «la Vérité » qu'une validité et une fécondité?

La théorie organisationnelle du cerveau, quand elle est appliquée de façon rigide, occulte en effet d'autres façons de comprendre et bloque systématiquement la prise en compte de certaines informations - et ce, non seulement à propos du développement du corps humain, mais à propos du développement en général. Même dans les études sur les petits mammifères, on sait depuis des décennies que si l'exposition précoce aux hormones stéroïdiennes affecte le développement du cerveau, ces effets hormonaux peuvent cependant être complètement annihilés ou outrepassés par la suite. La conviction que l'organisation cérébrale est permanente et qu'elle crée chez un animal des tendances latentes qui seront vraies pour toujours a empêché les scientifiques de prêter attention à certaines données dont ils et elles disposaient. Prenons l'expérience faite par le laboratoire de Roger Gorski, à UCLA en 1969, ayant mis en évidence que des rates qui avaient été exposées à des taux modérés de testostérone pendant les premiers jours de leur vie étaient moins réceptives aux tentatives de monte des mâles. Si on laissait ces femelles dites " androgénisées » s'habituer à leur cage pendant deux heures avant d'y mettre un mâle, les effets de cette exposition hormonale précoce disparaissaient complètement. Quelque chose qui peut être changé en deux heures de temps peut difficilement être qualifié d'effet permanent sur le cerveau. Mais le modèle était si fort, si puissant, qu'il a occulté cette information.

C'est vrai que je ne m'intéresse pas à «la Vérité » avec un V majuscule, car je crois qu'il n'y a pas de vérité sous-jacente et universelle de la sexualité humaine et du genre. Mais pour faire ce que je cherchais à faire avec ces études, qu'il y ait ou non une vérité universelle n'a pas d'importance. Je voulais seulement savoir, à propos des scientifiques utilisant la théorie organisationnelle du cerveau : qu'est-ce que la sexualité humaine, d'après ces personnes ? Comment est-ce qu'elles l'étudient ? Et l'étudient- 
elles d'une façon qui leur permet de soutenir les généralisations qu'elles prétendent pouvoir faire?

En lisant votre livre, on dirait que les personnes qui mènent ces recherches sont en quête d'essences cachées derrière les comportements et les corps ; quelque chose de stable, de défini une fois pour toutes, au-delà de la matérialité changeante qui est paradoxalement considérée comme «moins réelle » que ces essences.

Cette croyance en des essences devient parfois même plus importante que le phénomène observable. C'est ainsi que certain.e.s scientifiques ont traité la question des changements dans les normes de genre au cours du temps : en affirmant que ce ne sont pas les petits détails concrets du genre que les hormones façonnent (comme les camions ou les poupées), que c'est plus abstrait que cela. Par exemple, selon la thèse de Simon Baron-Cohen (Baron-Cohen, 2004), c'est l'attention dirigée vers les objets plutôt que vers les personnes. D'autres scientifiques ont même suggéré que nous ne devrions pas trop nous soucier de la variabilité des normes de genre, parce que le mécanisme réel est probablement que ces expositions précoces aux hormones orientent un individu vers ce qui est codé comme masculin ou féminin dans son monde à ce moment-là, quoi que ce soit. Cette idée est incroyable, car elle est si malléable qu'elle en devient non réfutable - et c'est difficile d'imaginer à quel mécanisme concret cela pourrait correspondre.

À un niveau très concret, la théorie de Baron-Cohen s'effondre complètement dès qu'on fait attention à ce qui constitue les catégories utilisées. Dans sa théorie, avoir un " cerveau masculin » signifie être orientéee vers les choses et les systèmes et avoir un «cerveau féminin » signifie être orientée vers les personnes. L'un des problèmes les plus importants de cette théorie est qu'elle contient beaucoup de catégorisations stéréotypées, certaines activités étant considérées comme «liées aux personnes » parce qu'elles sont majoritairement exercées par des femmes et d'autres étant «liées aux choses " parce qu'elles sont surtout exercées par des hommes. Par exemple, il classe les soins infirmiers comme témoignant d'une orientation vers les personnes, alors que pour être infirmièree, vous devez vous coordonner avec des systèmes multiples. Dans un hôpital, l'équipe infirmière doit avoir une compréhension très complexe et dynamique des systèmes impliqués : la pharmacologie, les horaires tournants en tout genre, les routines opératoires et post-opératoires... Ce que j'essaie de dire, c'est que les histoires abstraites s'écroulent souvent lorsqu'on observe attentivement à l'échelle des mécanismes. Des associations rapides comme "les soins infirmiers, c'est féminin », « l'ingénierie, c'est masculin » nous empêchent de nous demander ce qui entre précisément dans les catégories et comment on les définit.

Une des rares métaphores que vous utilisez dans le livre consiste à dire que le sexe, le genre et la sexualité sont un fil à trois brins (a three-ply yarn). Pourquoi cette image? 
J'aime cette métaphore car en anglais, elle a plusieurs sens. Premièrement, un fil est une fibre entortillée, et « à trois brins » signifie qu'il y a trois filaments. Lorsqu'ils sont tissés ensemble, ils semblent être une seule et même chose parce qu'ils sont fermement liés. Ils peuvent être démêlés d'une certaine façon, mais ils trouvent une grande partie de leur matière dans le fait d'être tressés ensemble. Sur le plan conceptuel, on peut séparer le sexe, le genre et la sexualité - c'est utile de les dissocier - mais sur le plan ontologique, ce n'est pas clair. Je ne suis pas en train de dire que dans la réalité, ils constituent des domaines objectivement séparés et je ne pense pas non plus qu'il s'agit de parties d'un domaine universel ou « objectivement réel » du sexe. Je pense que ce sont des constructions qui nous aident à comprendre comment nous divisons l'expérience humaine, que ce sont différentes façons d'appréhender des dimensions de la vie.

Deuxièmement, en anglais, le mot yarn signifie « histoire ». L'histoire que nous nous racontons à propos des relations entre le sexe, le genre et la sexualité, consiste à dire qu'ils font partie d'une seule et même chose et qu'ils devraient «naturellement » s'accorder d'une façon particulière. Cette façon particulière, c'est l'idéal hétéronormatif, qui considère que les corps qui ont certains types de parties devraient aller avec certains types de désirs pour d'autres corps avec d'autres types de parties et qu'ils devraient également aller avec un ensemble de façons d'être dans le monde - différents traits de caractère, différentes compétences, différents rôles... J'appelle également cela le «forfait tout compris » (package deal) du sexe, du genre et de la sexualité. Le fait que les scientifiques appréhendent de façon critique certaines formes de sexualité, alors que d'autres formes sont admises telles qu'elles sont, semble alors naturel. Qu'on attende des individus exposés à des hormones qui ne sont pas typiques de leur sexe génétique qu'ils aient des désirs ou des comportements «de l'autre sexe» (cross-sex), comme on dit, semble alors aussi assez naturel. Cela rend en apparence évident et naturel une grande partie de ce qui structure les recherches dans ce domaine et fait qu'il est difficile pour les chercheuses-eurs de voir à quels endroits les données expérimentales ou d'observation ne concordent pas avec les croyances populaires.

Vous écrivez que le genre n'est pas une façon très utile de classer les prédispositions des individus. Mais précisément, cette catégorie n'est-elle pas utile pour la médecine, par exemple? Le genre a des effets qui sont importants pour les diagnostics et les traitements. Ne serait-il pas alors intéressant de conduire des recherches à partir des différences de sexe et/ou de genre, de façon à rendre la pratique des médecins plus personnalisée?

Tout d'abord, l'approche médicale aujourd'hui fait une grave confusion entre le genre comme domaine social et les caractéristiques biologiques du corps liées au sexe. Il y a donc une tendance, même si le terme « genre » est utilisé, à élaborer presque toujours le raisonnement comme si toute différence observée venait de différences fondamentales et originelles entre les corps sexués. Deuxièmement, le genre produit as- 
surément des effets. Anne Fausto-Sterling a mené de magnifiques travaux sur la manière dont les façons genrées d'être dans le monde modèlent les corps, avec l'exemple des os et du développement osseux (Fausto-Sterling, 2005). Ce qui semble ainsi être une différence due au sexe est profondément lié au genre, et en faisant des comparaisons entre hommes et femmes de différents groupes et en observant les différentes façons dont le genre est performé dans ces groupes en particulier, on peut voir qu'il joue un rôle très important dans la façon dont les os se développent. En épidémiologie sociale, les travaux de Nancy Krieger (Krieger, 2003) sont également utiles sur cette question.

Mon souci avec l'utilité supposée du genre dans la médecine est que cette division habituelle des personnes entre hommes et femmes nous fait trop souvent perdre de vue les mécanismes spécifiques de la maladie ou affection qui nous intéresse. Il est très rare qu'il y ait un dimorphisme réel. Que ce soit pour les symptômes de la crise cardiaque, le cholestérol ou encore les réactions aux médicaments, la distribution des hommes et celle des femmes se recouvrent et il y a beaucoup de variabilité au sein de chaque sexe. Considérer trop vite que le sexe est un facteur important, voire le facteur principal, parce que cela semble pratique et facile à connaître nous conduirait à ne pas choisir le meilleur traitement dans de nombreux cas - par exemple, beaucoup d'hommes dans un groupe auront la forme dite «féminine » du cas (et inversement).

Enfin, la médecine sexospécifique est tout sauf un pas vers une médecine personnalisée. C'est, en revanche, une tactique marketing très efficace qui correspond bien à ce qui se produit depuis trente ans, à savoir que pratiquement toutes les industries ont découvert à quel point il était lucratif de diviser le marché par le genre. Elles sont capables d'augmenter significativement les ventes globales en divisant - qu'il s'agisse de l'Aspirine (on a une version pour femmes et une version pour hommes), ou des jouets (on a des frisbees roses et des bleus, même si c'est le même jouet). Ce ne sera jamais aussi lucratif de passer à une médecine personnalisée, en partie parce que la façon dont on devra diviser les groupes de patients dépendra des maladies, tandis que le sexe est une catégorie qui va rester cohérente et pour laquelle il est facile de concevoir un marketing ciblé. Je pense que nous devons être conscient·e-s que ce qui motive tout cela n'a pas grand chose à voir avec un progrès dans la façon de répondre vraiment aux caractéristiques corporelles individuelles.

Vous travaillez également sur la question de la "race» en biologie et en médecine, par exemple dans vos recherches actuelles sur la testostérone et les "tests de féminité » chez les athlètes (Karkazis, Jordan-Young, Davis ef Camporesi, 2012). Quels sont les points communs et les différences entre les questions de "race » et de genre dans ces domaines?

Tout d'abord, de plus en plus, l'une des tâches clefs dans mon travail consiste à garder à l'esprit que théoriquement et politiquement je dois toujours rendre justice au fait que ces types de différences (« race », genre, classe sociale, nationalité, sexualité...) 
ne sont pas des domaines parallèles qu'on peut comparer les uns aux autres, mais qu'ils sont imbriqués. C'est la raison pour laquelle il importe de les appréhender comme se constituant toujours mutuellement les uns les autres. Ce que cela implique pour le projet sur lequel je travaille actuellement, c'est que les idéaux particuliers concernant les corps féminins et masculins ont toujours une identité raciale implicite. Ils renvoient à un imaginaire racialiste et à des peurs à l'égard de certains types nationaux et des formes de féminité et de masculinité qui leur sont liées. Depuis 2011, des règles quant à la testostérone dans le sport établissent que les femmes dont le propre corps produit un niveau élevé de testostérone ne peuvent plus participer à la compétition en tant que femmes. Cela semble assez neutre, comme si cela pouvait concerner n'importe qui, mais il s'avère que les femmes qui sont affectées par ces règles sont toutes des femmes de couleur venant des pays du Sud (et d'une grande pauvreté, donc il y a également une composante économique). Il y a énormément de soupçons qui sont dirigés vers des femmes en particulier et beaucoup d'énergie est dépensée pour les poursuivre, celles dont certaines personnes pensent qu'elles pourraient avoir plus de testostérone dans le corps qu'elles ne devraient. Cela s'articule avec une racialisation de la féminité, la féminité elle-même étant codée implicitement comme blanche dans l'imaginaire occidental qui a élaboré ces mesures et les impose.

Par ailleurs, au niveau discursif, trouver le contenu racialiste de ces politiques est bien plus difficile que de trouver l'idéologie de genre. Les croyances à propos des corps féminins et masculins sont sous nos yeux, dans le texte explicite de ces politiques et des rapports scientifiques qui les soutiennent, tandis que le « discours de la race » est codé : on parle de nationalité, d'athlètes venant des pays du Sud, des pays en développement, etc. Notre défi dans cette recherche, à mon équipe et à moi, c'est de repérer et de rendre explicite la pensée racialiste sous-jacente qui modèle à la fois ces politiques elles-mêmes et la façon dont elles sont imposées.

Je suis enthousiaste quant à ce projet car, lorsque j'ai écrit Brain Storm, je savais qu'il y avait cette homogénéité artificielle de la masculinité et de la féminité dans cette science, et je soupçonnais que la plupart des groupes étudiés - assemblés pour créer les normes à l'aune desquelles tout le monde serait mesuré - étaient constitués de personnes blanches, de classe moyenne, vivant aux États-Unis ou en Europe de l'Ouest. Mais je ne savais pas comment exposer de façon analytique cette incapacité à problématiser la norme blanche. Je suis à présent en train de gagner une force méthodologique sur la façon dont les pratiques concrètes dans ces sciences du genre et du sexe font de la féminité blanche - un idéal de féminité blanche, puisque la féminité blanche elle-même n'existe pas - la féminité éternelle et unique. Apprendre des méthodes pour repérer comment et où la blanchité (whiteness) est présente et comment cela se construit est nouveau pour moi et je suis ravie de pouvoir le faire. 


\section{Bibliographie}

Barad K. (1998), « Getting real: Technoscientific practices and the materialization of reality ", Differences, 10(2), pp. 87-126.

Baron-Cohen S. (2004), The essential difference, Londres : Penguin.

Fausto-Sterling A. (2005), " The bare bones of sex: Part 1 - sex and gender », Signs, 30(2), pp. 1491-1527.

Haraway D. (1998), « Situated knowledges: The science question in feminism and the privilege of partial perspective », Feminist studies, 14(3), pp. 575-599.

Harding S. (1992), "Rethinking Standpoint Epistemology: What is 'Strong Objectivity?», The Centennial Review, 36(3), pp. 437-470.

Jordan-Young R. (2010), Brain Storm. The Flaws in the Science of Sex Differences, Cambridge : Harvard University Press.

Karkazis K., Jordan-Young R., Davis G. \& Camporesi S. (2012), «Out of bounds ? A critique of the new policies on hyperandrogenism in elite female athletes », The American Journal of Bioethics, 12(7), pp. 3-16.

Krieger N. (2003), «Genders, sexes, and health: what are the connections-and why does it matter ?», International journal of epidemiology, 32(4), pp. 652-657.

Latour B., Woolgar S. (1998), La vie de laboratoire, Paris : La Découverte.

Longino H. (1990), Science as social knowledge. Values and objectivity in scientific inquiry, Princeton : Princeton University Press.

Longino H. (2013), Studying human behavior. How scientists investigate aggression and sexuality, Chicago : University of Chicago Press.

Mol A. (2002), The body multiple. Ontology in medical practice, Durham: Duke University Press. 